A N N A L E S Annales de Bretagne et des Pays de l'Ouest

\title{
Les cités sardinières, portes ouvertes aux épidémies de choléra (1852-1914)
}

Sardine processing and the dangers of cholera epidemics (1852-1914)

Jean-Christophe Fichou

\section{(2) OpenEdition}

Édition électronique

URL : http://journals.openedition.org/abpo/3727

DOI : $10.4000 / a b p o .3727$

ISBN : 978-2-7535-7411-3

ISSN : 2108-6443

\section{Éditeur}

Presses universitaires de Rennes

\section{Édition imprimée}

Date de publication : 20 décembre 2017

Pagination : 133-153

ISBN : 978-2-7535-7409-0

ISSN : 0399-0826

\section{Référence électronique}

Jean-Christophe Fichou, «Les cités sardinières, portes ouvertes aux épidémies de choléra

(1852-1914) », Annales de Bretagne et des Pays de l'Ouest [En ligne], 124-4 | 2017, mis en ligne le 20

décembre 2019, consulté le 03 janvier 2020. URL : http://journals.openedition.org/abpo/3727 ; DOI :

10.4000/abpo.3727 


\title{
Les cités sardinières, portes ouvertes aux épidémies de choléra (1852-1914)
}

\author{
Jean-Christophe FicHOU \\ Docteur en histoire, Lycée de Lapérouse-Kérichen (Brest)
}

Si la France du XIX ${ }^{e}$ siècle n'est plus la proie des grands fléaux épidémiques du passé, comme la grande peste du $\mathrm{Xv}^{\mathrm{e}}$ siècle qui anéantit le tiers de la population européenne, elle reste toutefois ouverte aux pandémies, notamment celles de choléra. Même si la plus meurtrière d'entre elles emporte dix fois moins de victimes qu'une peste médiévale ${ }^{1}$, les décès restent importants, notamment dans les villes nouvelles nées de l'industrialisation, et plus particulièrement dans les cités portuaires bretonnes et vendéennes dont l'essor est dû à l'implantation des conserveries de poisson, des cités ouvertes sur le monde, lieux d'accueil favorables aux épidémies. Cette activité est née vers 1825 et elle a connu très vite un formidable essor, après 1855, en remportant l'assentiment des gastronomes et de la grande bourgeoisie $^{2}$. Dès les années 1880, on compte plus de 150 usines réparties entre Camaret et La Rochelle, dont plus de la moitié dans le seul département du Finistère, et le nombre de pêcheurs et d'ouvrières de "friteries " ne cesse d'augmenter. Tous les ports sardiniers sont caractérisés au XIX ${ }^{\mathrm{e}}$ siècle par une croissance démographique exceptionnelle, mais aussi par une hygiène publique et individuelle déplorable, résultat de la convergence entre les intérêts des usiniers, le laxisme des conseils municipaux et les comportements individuels indifférents et passifs. Dans ces conditions, il n'est pas surprenant de constater que les villes ayant développé la conserverie de poisson sont les premières et les plus durement touchées par les épidémies de choléra qui déciment les populations de ces nouveaux foyers industriels tout au long du XIX ${ }^{\mathrm{e}}$ siècle. La Bretagne intérieure est beaucoup moins touchée, il devient donc évident que le fléau cholérique se transmet

1. LÉONARD, Jacques, La France médicale au XIX siècle, Paris, Gallimard, coll. Archives, 1978, p. 152.

2. Rapport du jury international. Exposition Universelle de Paris 1867, CHEVALIER, Michel (dir.), Ministère du Commerce et de l'Industrie, Paris, imp. Paul Dupont, 13 volumes, 1868. Tome onzième, Groupe VII, aliments frais et conservés : classe 70 , sardines à l'huile et thon mariné, p. 169. 
par la mer et se propage sur un littoral très peuplé, à la convergence de tous les trafics du cabotage européen, d'hébergement de pêcheurs tous les jours plus nombreux et d'accueil de marins militaires de retour de campagnes coloniales. Toute cette population s'entasse pauvrement dans des quartiers construits sans plan, sans hygiène, sans égout, sans eau potable bien souvent. Parmi ces familles d'ouvriers et de pêcheurs, affaiblies sans doute il est vrai par l'alcoolisme, les dégâts des épidémies sont immédiats et virulents. Si pour beaucoup de médecins c'est " le gros vin bleu et surtout la mauvaise eau-de-vie, le grand fléau qui vient ronger le peuple ${ }^{3}$ ", l'absence de mesures protectrices dans les petits ports favorise l'introduction de toutes les maladies transmissibles par la voie maritime; les cités sardinières n'échappent pas à la règle, bien au contraire.

Tous les éléments sont réunis pour que les épidémies de choléra se diffusent comme des traînées de poudre et soient particulièrement meurtrières dans les ports sardiniers bretons jusqu'en 1895, date à laquelle les autorités, et notamment le ministère de la Marine, décident de combattre ce mal avec des moyens d'envergure.

\section{Des villes nouvelles et sales}

La crasse des villes et des usines, la surpopulation urbaine, le manque d'eau potable sont responsables en grande partie de la multiplication des épidémies d'une rare violence et notamment des attaques répétées de la variole, du typhus, transmis par les poux, de la typhoïde, de la dysenterie et du choléra, transmis par les eaux sales, qui ravagent la Bretagne littorale, "le conservatoire du typhus en France " ". À cela deux causes : "Les habitudes nomades des côtes où presque tous les habitants exercent la profession de marins [et] les migrations incessantes des filles de la campagne vers la ville ${ }^{5 . "}$

\section{Un littoral industriel urbanisé}

Comme tous les bassins industriels européens au XIX ${ }^{\mathrm{e}}$ siècle, le littoral sardinier connaît une croissance démographique exceptionnelle, surtout par rapport aux régions environnantes, intérieures et agricoles, de la Bretagne ou de la Vendée. Après 1850, dans certaines localités, comme Douarnenez, Le Guilvinec, Concarneau, La Turballe ou Croix-de-Vie, la population urbaine triple en moins d'un demi-siècle. On assiste dans ces cités sardinières à une véritable explosion démographique. Les conserve-

3. CARADEC, Louis, Topographie médico-hygiénique du département du Finistère, Brest, 1860 , p. 88.

4. Henri Monod, premier directeur de l'Assistance et de l'Hygiène (1887-1905). Cité dans Murard, Lion et ZYLBERMAN, Patrick, L'Hygiène dans la République. La santé publique en France, ou l'utopie contrariée 1870-1918. Paris, Fayard, 1996, p. 196.

5. MarTin, Eugène, Villages et fermes dans le département du Finistère, leur hygiène, Thèse pour le doctorat en médecine, Bordeaux, 1913, 54 p. 
ries forment la trame des réseaux urbains et la ville se construit en fonction de leur installation, aux abords immédiats des quais, déroulant " un véritable front pionnier marqué par un semis de villes-champignons ${ }^{6}$ ". Dans ces villes à la croissance anarchique, les usines et le port sont encerclés par un habitat désordonné. L'horizon ne s'ouvre que sur la mer pour des ouvrières et des pêcheurs vivant dans un environnement dégradé, d'une saleté épouvantable. Dans toutes les villes sardinières, la surmortalité est une composante de la spécificité démographique urbaine ${ }^{7}$. Elle est fondée avant tout sur la pauvreté, comme le souligne le célèbre médecin de la Marine Fonssagrivres : " Entre toutes les maladies chroniques qui rongent le corps social, la misère est certainement une des plus hideuses, des plus invétérées, peut-être même des moins curables ${ }^{8}$. " Ce qui n'empêche pas le gonflement démographique des centres sardiniers. Dans le Finistère, l'arrondissement de Quimper connaît la plus forte progression et passe de 80974 habitants en 1856 à 203581 en 1911. Pour tous les observateurs, cet accroissement exceptionnel s'explique surtout par le développement des petits ports de pêche sur les côtes des cantons de Pont-l'Abbé, PontCroix et Douarnenez où la population cantonale passe de 9996 habitants en 1800 à 33143 en 1911. Ainsi, " le sordide village de Tréboul est devenu une petite ville [...]. L'ancienne ville morte de Port-Louis s'est ranimée avec l'industrie sardinière ${ }^{9}$ ". Dans le Morbihan, le phénomène est tout aussi caractéristique.

Dans ces conditions, les foyers épidémiques n'épargnent jamais la population agglomérée des ouvriers et des matelots, toujours la première et la plus touchée. Les fortes concentrations humaines et les conditions de vie sommaires dans des quartiers où l'entassement, l'insalubrité et la promiscuité sont la règle, expliquent l'importance des dégâts, d'autant plus que l'eau est un bien rare, partagé par une population et des industriels toujours plus nombreux. De plus, l'habitude séculaire de s'en remettre aux pouvoirs salvateurs de Notre-Dame-du-Bon-Secours diffère le concours, vécu comme une intrusion, des médecins qui interviennent trop tard.

Quant à la salubrité publique, il convient de combattre de front les diarrhées infantiles, la malnutrition, la sous-nutrition, le manque d'hygiène la plus élémentaire, les eaux souillées... Sans réseau d'adduction d'eau potable, ni d'évacuation des eaux usées, sans rue pavée, sans mesure coer-

6. Coulıou, Jean-René, La Pêche bretonne, Rennes, Presses Universitaires de Rennes, 2002, p. 141.

7. BAIROCH, Paul, De Jéricho à Mexico, villes et économies dans l'histoire, Paris, Gallimard, 1985, p. 298.

8. FonsSAgrives, Jean-Baptiste, "Des obstacles que rencontre l'hygiène ", dans Montpellier médical, décembre 1866, p. 4. Professeur à l'École de médecine navale de Brest, puis à la faculté de médecine de Montpellier, il meurt en 1884 à Crach, dans le Morbihan, en soignant la population des environs d'Auray et de Lorient où sévit alors une épidémie de choléra.

9. Vallaux, Camille, La Basse Bretagne. Étude de géographie humaine, Genève/Paris, Slatkine, 1980 (1905), p. 137. 
citive, sans personnel de surveillance, dans des villes sales et enfumées, sans plan d'urbanisation, où s'entasse une population pauvre, les municipalités apparaissent totalement dépassées par les événements. Les interventions locales ou celles de l'État sont toujours trop tardives. La prise de conscience est réelle mais progressive, dispersée et lente en raison du principe fondamental de la liberté individuelle d'entreprendre et de la crainte d'un État trop présent. Les initiatives sont, de toute manière, trop rares pour engendrer un vaste mouvement d'application de règles sanitaires évidentes et nécessaires. C'est bien une " permanence de l'échec ${ }^{10}$ " qui caractérise toutes les tentatives faites pour améliorer l'hygiène de la vie urbaine dans des cités surpeuplées et sales, comme celles du littoral sardinier. Les taux de mortalité bretons restent supérieurs à la moyenne nationale jusqu'en 1911; ils sont particulièrement élevés dans toutes les villes sardinières jusqu'en 1895 environ, toujours supérieurs aux moyennes départementales du Finistère et du Morbihan. Ces chiffres sont dus à une surmortalité adulte qui s'explique par les progrès de l'alcoolisme, les dangers de la pêche, et l'insuffisance criante des équipements sanitaires ${ }^{11}$.

\section{Les villes sardinières, les portes de l'épidémie}

"Les épidémies sont fréquentes partout, [...], où le mépris de l'hygiène est une règle générale : mais ce sont surtout sur l'Ar-Mor des épidémies localisées de choléra [...]. Ces épidémies éclatent périodiquement dans les centres sardiniers comme Tréboul, Penmarc'h, Le Guilvinec ${ }^{12}$. "

Pour tous les observateurs il est évident que les épidémies de choléra arrivent en France par les ports, accès privilégiés du fléau. Le constat est ancien. Une police sanitaire du littoral, sans grands moyens il est vrai, se met en place dès le début des années 1820 " pour prévenir l'introduction en France des maladies contagieuses ${ }^{13}$ ». Le 21 août 1824 est publiée en France la première circulaire prescrivant aux préfets des départements maritimes et frontaliers les mesures à prendre en cas "d'invasion " du choléra ${ }^{14}$. Il est notamment prévu dans les ports militaires comme à Brest et à Lorient de surveiller les bateaux suspects et de les isoler en quarantaine si les préfets maritimes conçoivent le moindre doute de contagion.

"Les navires suspects (art. 36 du règlement général) ne sont admis à libre pratique qu'après une observation de vingt-quatre heures dans l'isolement et une inspection médicale ayant permis de constater l'absence d'accidents cholériques à bord ${ }^{15}$."

10. Murard, Lion et Zylberman, Patrick, L'Hygiène dans la République..., op. cit., p. 366.

11. MEYER, Jean, " D’une révolution manquée à une révolution possible ", dans DELUMEAU, Jean (dir.), Histoire de la Bretagne, Toulouse, Privat, 1969, p. 465.

12. VallauX, Camille, La Basse-Bretagne..., op. cit., p. 309.

13. Arch. dép. des Côtes-d'Armor, 5 M 7, police sanitaire, An IX-1850.

14. Arch. dép. du Finistère, 5 M 48, Paris, le 21 août 1824.

15. Règlements contre le choléra; mesures sanitaires applicables aux provenances de choléra dans les ports de la Manche et de l'Océan, sl, nd. 
Pour confirmer cette attitude de prudence, deux lazarets sont mis en construction en 1825 sur l'île de Trébéron dans la rade de Brest et sur l'île Saint-Michel dans la rivière de Lorient, mais on en reste là ${ }^{16}$. Une épidémie de fièvre jaune qui se déclare à Gibraltar en 1828 aurait dû ranimer le zèle des promoteurs du premier système de veille et de cordon sanitaire maritime, d'autant plus que la menace cholérique se précise. En 1831, des mesures sont prises par le gouvernement de Louis-Philippe, notamment pour tenter de protéger les ports et les préfets concernés sont avertis :

"Messieurs, on a remarqué que des navires partis d'un port infecté du choléra morbus, sans que leurs équipages parussent atteints de cette maladie, en ont éprouvé l'irruption le jour ou le lendemain de leur appareillage; et que, conséquemment, leur état sanitaire est mieux indiqué par une patente de santé délivrée ou visée aux lieux de relâche que par celles qui sont prises aux lieux de départ ${ }^{17}$."

Le 20 septembre 1831, une ordonnance royale impose l'établissement d'une Intendance sanitaire au chef-lieu des départements maritimes afin de centraliser les informations et de donner des ordres clairs pour tenter de combattre l'éventuelle épidémie ${ }^{18}$. Le 19 novembre $1831^{19}$, le danger se précise sur les côtes bretonnes et les préfets concernés sont alertés après l'apparition du choléra sur les côtes anglaises, à Sunderland. En conséquence, obligation est faite aux navires français et étrangers sortant du Sund, en provenance de la Baltique où sévit l'épidémie, et à destination de la France, de prendre une patente de santé auprès du consul de France à Elseneur. Mais ces mesures sont insuffisantes et ne freinent pas ce qui sera la première pandémie de choléra, au sens littéral du terme, c'est-àdire mondial. Le choléra, maladie jusqu'alors éminemment exotique, est signalé pour la première fois en France, à Calais, le 15 mars $1832^{20}$. Ensuite il se propage jusqu'à Paris et dans toute la Manche par voie maritime, si bien que le ministre de la Marine, le comte d'Argout, impose la quarantaine à tous les bateaux en provenance de l'Europe du Nord. Au début du mois d'avril, le préfet du Finistère écrit au maire de Quimper afin de l'inciter à prendre un arrêté pour prescrire tous les moyens utiles d'assainissement et de propreté, notamment dans les quartiers intra-muros où s'entassent des amas considérables de fumiers. Le 3 avril, l'évêque de Quimper promet la mise à disposition des bâtiments du grand séminaire pour servir d'hôpital pour le cas où le choléra se manifesterait dans l'en-

16. Arch. dép. des Côtes-d'Armor, 5 M 10; il était projeté de construire un lazaret à SaintBrieuc, mais le projet est abandonné.

17. Arch. dép. du Finistère, 5 M 48, Paris, le 9 septembre 1831, le ministre du Commerce et des Travaux publics.

18. Arch. dép. des Côtes-d'Armor, 5 M 8, Service sanitaire du littoral.

19. Paris, le 19 novembre 1831, le conseiller d'État, vice-président du Conseil de Santé, Hély d'Oissel au préfet du Morbihan. Cité dans MAURICET, Alphonse, Exposé historique des épidémies de choléra asiatique, Vannes, imprimerie Galles, 1884.

20. Bourdelais, Patrice et Raulot, Jean-Yves, Une peur bleue. Histoire du choléra en France, 1832-1854, Paris, Payot, 1987, p. 79-82. 
ceinte de la cité épiscopale. Le 18 avril, le baron de Las Cases adresse au maire de Quimper, depuis Paris, une instruction à distribuer aux malades cholériques sur les prescriptions médicales jugées utiles par de nombreux médecins qui n'ont jamais été confrontés au choléra. Des consignes en français, mais aussi en breton, sont imprimées sur les moyens de lutter contre la maladie ou de s'en prémunir ${ }^{21}$. C'est bien par la mer que le choléra arrive en Bretagne où le premier cas est signalé dans le Finistère, à Quimper, le 10 mai. Il s'agit d'un certain Catel, maître de manœuvre sur la corvette Eglé de la Royale, partie de Toulon le 17 avril précédent; il décède le 11 mai $1832^{22}$. Son corps est autopsié sans aucune précaution et enseveli le lendemain matin. On dilue un peu de son sang dans de l'eau donnée à boire à des canards qui, selon les médecins, " n'en éprouvent aucun dérangement ". Le même jour, deux femmes de l'hospice présentent les symptômes de la maladie alors que le docteur de Kergaradec écrit encore « il n'y a aucune connexion entre ces deux faits et celui du matelot Catel ». De Quimper, le choléra se répand dans le nord et l'ouest du département, à Douarnenez, puis à Brest, à Morlaix et dans les Côtes-du-Nord, à Paimpol. Le 24 août 1832, on déplore le premier décès à l'île Molène où le choléra a été apporté par un pilote de Brest. Dans le Morbihan, le premier décès est enregistré le $22^{23}$ ou le 23 juin 1832. Il s'agit de Pierre Chauvel, capitaine du sloop Le Georges, arrivé le 20 juin à Lorient en provenance de Saint-Malo, après des escales à Brest et Quimper, "pays infestés ${ }^{24}$ ". Au cours de l'été, on combat le choléra à Lorient, à Port-Louis, à Gâvres, à Étel, à Groix et sur tout le littoral jusqu'au Croisic et à Batz-sur-Mer où il est signalé dès la fin du mois de juillet. Dans le nord de la région, il s'étend aussi ; il est à Paimpol le 4 août ${ }^{25}$. En octobre, il reparaît dans le Morbihan, à Belz et à Belle-Île, puis dans toute la région de Lorient; en novembre, on le retrouve à Concarneau, à Saint-Pol-de-Léon et à $\operatorname{Roscoff}^{26}$.

Le doute n'est plus possible, il s'agit bien d'une maladie contagieuse et non plus infectieuse comme on l'imaginait encore, mais il faut attendre 1882 et la découverte du bacille de la tuberculose, et l'année suivante celle du vibrion cholérique, pour avoir raison du dernier carré des sectateurs

21. Arch. mun. de Quimper. LeJUMEAU de KERGARADEC, Jean, Alexandre, Quelques mots sur le choléra-morbus épidémique et les moyens de s'en prévenir, Quimper, 1832.

22. Arch. mun. de Brest, 2 A 2 87. Citée dans LE NAOURES, Jacques, Les épidémies de choléra dans le Finistère au XIX siècle, Brest, Université de Bretagne Occidentale, mémoire de maîtrise, 1995, p. 11.

23. LE BouËDEc, Gérard, Le Port et l'Arsenal de Lorient. De la Compagnie des Indes à la Marine cuirassée. Une reconversion réussie (XVIII -XIX ${ }^{e}$ siècle), Paris, Librairie de l'Inde, 1995 , p. 725.

24. Lorient, le $1^{\mathrm{er}}$ novembre 1832, le sous-préfet Eugène Mancel. Cité dans MAURICET, Alphonse, Exposé historique et statistique des épidémies de choléra asiatique, Vannes, imp. Galle, 1884, p. 8.

25. KerlÉVÉo, Jean, Paimpol au temps d'Islande, Douarnenez, le Chasse-Marée, 1998 (1944), p. 65.

26. Colet, Daniel, " Le Choléra de 1832 dans le Finistère ", Bulletin de la société archéologique du Finistère, t. 113, 1984, p. 237-270. 
anti-contagionnistes ${ }^{27}$. La maladie se déplace avec les hommes et particulièrement vite par la mer sans que l'on connaisse réellement les chemins utilisés. En revanche, on constate rapidement qu'elle frappe surtout en milieu urbain les personnes âgées et les nourrissons, et qu'elle sévit dans les quartiers les plus pauvres. On ne sait pas non plus pourquoi elle s'éteint, bien que certaines conclusions s'imposent. À Port-Navalo, l'épidémie disparaît le 18 novembre 1832 : « [E]lle s'est éteinte quand elle n'a plus trouvé sa proie d'affection, la misère ${ }^{28}$."

En 1834, l'épidémie reprend après quelques mois de répit et touche le port de Brest à la fin du mois de juillet; le 12 août, les habitants de Vannes sont contaminés puis, le 3 septembre ceux de Lorient - parmi lesquels on dénombre 153 morts. Elle se propage ensuite à Groix, à BelleÎle et Locmariaquer : " [C] omme on le voit, le littoral de l'Océan paie le plus large tribut ${ }^{29}$. "En août et septembre, les communes du Croisic et de Batz puis celles de Guérande et de Saint-Nazaire en Loire-inférieure sont aussi touchées. Le choléra atteint le Cap-Sizun à la fin de l'année 1834 : 3 décès à Pont-Croix en décembre, 11 décès à Cléden, 64 décès à Audierne du 25 décembre 1834 au 21 février $1835^{30}$. Dans le Finistère, c'est la commune littorale de Camaret qui paie le plus lourd tribut puisque l'on compte 356 décès, soit $1 / 7^{\mathrm{e}}$ de la population totale ${ }^{31}$ ! L'épidémie s'étend aussi sur le littoral nord de la Bretagne, toujours propagée par voie maritime. Au mois d'août, elle est attestée dans les Côtes-du-Nord, à Saint-Cast-le-Guildo, où officie le médecin Pierre Sébillot ${ }^{32}$, qui fait l'objet d'une citation pour son dévouement pendant l'épidémie.

\section{Le choléra omniprésent}

Après une décennie de latence, le mal frappe de nouveau. Le choléra est à Erdeven au début du mois de février 1849; le 17 février l'épidémie touche Lorient où elle entraîne 365 habitants dans la mort. À la veille de l'été, on pense qu'elle est enrayée, mais elle reprend dès l'automne. Elle commence le 21 septembre 1849 à Brest, d'où elle s'étend vers le nordouest; on l'identifie à Bodilis le 23 septembre et elle se propage ensuite dans tout le département puisqu'elle atteint Douarnenez le 26 septembre, au moment où la pêche à la sardine bat son plein. Le préfet s'en inquiète : " [C]'est à faire trembler pour Douarnenez qui a en ce moment une popu-

27. LAGET, Pierre Louis, "Les Lazarets et l'émergence de nouvelles maladies pestilentielles au XIX et au début du Xx ${ }^{\mathrm{e}}$ siècle ", In Situ, 2002-2, p. 2.

28. Port-Navalo, le 18 novembre 1832, le lieutenant des Douanes Le François Cité dans MAURICET, Alphonse, Exposé historique... op. cit., p. 18.

29. Ibidem, p. 19.

30. Heuté, Claude, Épidémies dans le Cap-Sizun au XIXe siècle, thèse de médecine, Université de Brest, 1985, 455 p.

31. Arch. dép. du Finistère, 5 M 48.

32. Il s'agit du père de Paul Sébillot, né à Matignon (Côtes-du-Nord) le 6 février 1843 et mort à Paris, le 23 avril 1918, ethnologue, écrivain et peintre français, 
lation réunie pour la pêche à la sardine ${ }^{33}$. " Locquénolé est atteint le 25 octobre, puis Morlaix quelques jours plus tard; on la retrouve à l'île de Sein où le premier cas est signalé le 30 novembre, attribué à la venue d'un maçon arrivé deux jours auparavant de Brest où l'épidémie fait des ravages. Après avoir entraîné la mort de 18 îliens, l'épidémie achève son voyage en décembre à Audierne et à Primelin. Dès les premiers symptômes, le préfet du Finistère avertit immédiatement son homologue du Morbihan pour qu'il puisse prendre des mesures de surveillance à l'entrée des ports de son département ${ }^{34}$. Mais toutes les précautions sont inutiles car l'épidémie reprend dans le Morbihan, avec moins de virulence qu'au printemps il est vrai.

Lors de l'épidémie de 1854, le premier cas breton est signalé dans le port de Morlaix au début de l'été, le 5 juillet. L'épidémie est à Vannes le 30 juillet; elle décline en septembre pour s'éteindre dans la ville alors qu'elle est signalée à Groix, où l'on dénombre 77 décès en novembre, et à Brest où le premier décès est constaté le 18 novembre ${ }^{35}$. Le ministère de la Marine s'inquiète de la tournure des événements et réclame des mesures fortes pour enrayer et éradiquer cette maladie qui continue de causer des ravages dans les populations littorales. Comme les autres nations européennes, et plus particulièrement les nations maritimes, connaissent les mêmes difficultés, une conférence sanitaire internationale ${ }^{36}$ est réunie en 1859 pour convenir d'actions communes pour alerter les autorités portuaires en cas de déclenchement d'une épidémie.

Il faut croire que les mesures décidées sont insuffisantes car l'épidémie de 1865-1866 s'avère la plus sévère pour les populations maritimes bretonnes. En 1865, la Stella, partie d'Alexandrie d'Égypte le $1^{\mathrm{er}}$ juin, arrive à Marseille le 11 juin en apportant dans ses soutes le choléra. Le mal sévit à Paris au cours de l'été et à Caen au début du mois d'octobre 1865. Le premier port touché en Bretagne est celui de Brest, mais il semble que dans ce cas le choléra nous soit parvenu par voie terrestre, sans doute ferroviaire, avec " un conscrit parti de Strasbourg, s'arrêtant à Paris, y puisant le germe du mal et venant mourir le 7 octobre très promptement à l'hôpital de la Marine ${ }^{37}$ ". Cependant les avis sont partagés dans le corps médical et certains médecins penchent plutôt pour un matelot du transport de l'État, L'Île de Ré venant de Lorient, ou pour un novice du vaisseau La Bretagne stationné en rade de Brest. Le premier décès morbihannais est quant à lui enregistré le 15 octobre à Lorient. Toujours est-il que la progression de cette épidémie est très rapide et particulièrement désastreuse pour la population des petits ports sardiniers. Ceux de Moëlan et

33. Arch. dép. du Finistère, 5 M 50, Quimper, le 3 octobre 1849 le préfet Brissot-Thivars. 34. Arch. dép. du Finistère, 5 M 49, Quimper, le 2 octobre 1849, le préfet du Finistère au préfet du Morbihan.

35. Arch. dép. du Finistère, 5 M 51.

36. Ministère des affaires étrangères, Protocoles de la conférence sanitaire internationale ouverte à Paris le 9 avril 1859, Paris, Imprimerie impériale, 1859, multipagination.

37. CARADEC, Théophile, Le Choléra de Brest en 1866, Paris, Germer-Baillière, 1866, 38 p. 
Riec sont touchés dès la fin du mois d'octobre, ce qui tend à confirmer l'origine lorientaise du mal. Le port de Lanvéoc, dans la rade de Brest, enterre son premier mort en février $1866^{38}$ puis les villes d'Audierne, du Guilvinec et de Douarnenez sont atteintes à leur tour; à Audierne, par exemple, 59 décès sont déclarés le 10 février. Deux mois plus tard, le 21 avril 1866, le commissaire de l'Inscription maritime, le secrétaire général de la Préfecture et le médecin départemental des épidémies sont au Guilvinec où vient de se déclarer de manière " foudroyante " l'épidémie parmi les pêcheurs des 200 à 300 bateaux de Douarnenez, de Concarneau et d'Audierne, soit environ 1200 marins qui pratiquent alors la pêche au maquereau dans les parages; pour la nuit, ils campent sur les rivages ou s'entassent dans des pièces sordides louées par la population locale, sans aucune précaution sanitaire.

"À notre arrivée nous avons trouvé une terreur générale répandue dans la population de ce petit port; ceux qui avaient des parents ou des amis en dehors de la localité déménageaient et abandonnaient leurs maisons en refusant d'enterrer leurs morts. Quelques mesures d'hygiène étaient nécessaires pour empêcher qu'on jetât sur la grève des cadavres de gros poissons qui étaient là en putréfaction. J'ai engagé les marins [...] pour recouvrir de sable les nombreux tas de goémons faisant fumier devant presque toutes les maisons. Enfin je leur ai conseillé de boire un peu moins ${ }^{39}$."

Nous l'avons dit, l'entassement dans des quartiers surpeuplés et malsains n'arrange rien, et le choléra, la variole ou la dysenterie y règnent en maître dès qu'ils se déclarent :

" Si cette maladie [le choléra] maintient son cours, on doit l'attribuer à l'imprudence et au manque de précaution de la plupart des malades ou à l'usage qu'ils font d'aliments malsains et même au défaut d'aliments nécessaires à leur existence, comme au manque de vêtements et de logements convenables car jusqu'à ce jour, cette maladie n'a sévi que contre la classe malheureuse ${ }^{40}$."

Cette terrible épidémie de 1865-1866 coûte la vie à 78 Concarnois en moins d'un mois. Elle entraîne la mort de 118 personnes dans tout le pays bigouden ${ }^{41}$ puis elle progresse, toujours par la mer, vers le nord du département : elle arrive en octobre 1866 dans les Côtes-du-Nord, où le premier cas est reconnu. En février 1867, malgré toutes les précautions prises, on recense le premier mort à Iffiniac à quelques kilomètres de Saint-Brieuc ${ }^{42}$. Encore une fois, les arrondissements du littoral paient le plus lourd tri-

38. Service Historique de la Défense, Marine Brest, MSS Supplément 49.

39. Service Historique de la Défense, Marine Brest, 3 P 2-3, Quimper, le 21 avril 1866, le commissaire de l'Inscription maritime au préfet.

40. Concarneau, le 11 février 1866, cité dans LE FLoc'H, Régis, Concarneau de 1852 à 1914 , Brest, Université de Bretagne Occidentale, Mémoire de maîtrise d'histoire, 1993, p. 83.

41. LE FloCH, Vincent, "Les Épidémies dans le pays bigouden, 1830-1914 ", dans les Actes du colloque, Le pays bigouden à la croisée des chemins, Université de Bretagne Occidentale, Bannalec, imp. régionale, 1993, p. 111-123.

42. Service Historique de la Défense, Marine Brest, MSS Supplément 49. 
but au choléra : 438 décès sur 534 cas déclarés dans le Morbihan, plus de 1100 sur 1316 dans le Finistère ${ }^{43}$.

Après vingt ans de répit, le 18 septembre 1885, un nouveau cas est signalé à Concarneau; c'est un marin de thonier en pêche au large de l'Espagne qui l'aurait apporté après avoir fait escale sur les côtes de Galice où sévissait alors une violente épidémie qui causa plus de 20000 morts en juillet et août ${ }^{44}$. Pour d'autres médecins, il semble que les éléments contaminateurs soient plutôt des membres du corps expéditionnaire de retour de Cochinchine et du Tonkin où une terrible épidémie de choléra a poussé les autorités de la Marine à de nombreux rapatriements de soldats et de marins touchés ${ }^{45}$. Plus de 720 passagers et les équipages de l'Annamite, du Finistère et du Rhin débarquent effectivement à Brest, porteurs pour certains, du vibrion mortel ${ }^{46}$.

"Une épidémie, à peine éteinte aujourd'hui, vient de sévir sur un certain nombre de communes du département. L'approche du printemps donne lieu de craindre le retour de ce fléau. Cette crainte grandit quand on songe que c'est précisément à cette époque qu'ont lieu dans certains ports de grandes agglomérations de pêcheurs. Il dépend en très grande partie des habitants de rendre cette crainte illusoire ${ }^{47}$."

Quoi qu'il en soit, tous les ports thoniers et sardiniers de Bretagne méridionale sont touchés à leur tour : Le Guilvinec, Audierne ${ }^{48}$, Douarnenez puis Brest au nord et enfin l'île de Sein. Sur l'île, les 24 cadavres sont enterrés à l'écart du cimetière communal, recouverts de chaux vive pour éviter une trop grande propagation de l'épidémie ${ }^{49}$. Vers le sud, Doëlan et Étel sont touchés à leur tour, puis Lorient et Groix ${ }^{50}$. C'est le port d'Audierne qui paie le plus lourd tribut et détient le record de cas déclarés (420) et du nombre de décès enregistrés (144). Ce taux de mortalité si élevé est expliqué dans un rapport du docteur Hébert, médecin des épidémies du canton de PontCroix : " Entre les deux quais, la ville est habitée par les commerçants, classe relativement aisée qui, grâce à ce bien-être, n'a pas eu à souffrir des atteintes du choléra... " alors que dans la Grand-Rue, habitée par les marins et leur famille,

43. Il s'agit des chiffres officiels, mais il semble bien que le nombre total des décès soit plus important.

44. Hamon, Augustin, Chronique de l'hygiène en Europe, Montréal, 1886, Daniel imprimeur, 56 p.

45. Pluchon, Pierre, Histoire des médecins et pharmaciens de Marine et des Colonies, Toulouse, Privat, 1985, p. 178.

46. Monod, Henri, Le Choléra. Histoire d'une épidémie. Finistère 1885-1886, Paris, Delagrave, 1892, p. 51.

47. Arch. dép. Finistère, 5 M 56, Quimper, le 10 février 1886, le préfet du Finistère.

48. Le 16 octobre, une cinquantaine de bateaux de pêche du Guilvinec surpris par la tempête trouvent refuge à Audierne. Neuf jours plus tard le premier cas de choléra est déclaré.

49. Service Historique de la Défense, Marine Brest, Manuscrit santé nº 27, rapport du médecin Louis Gouzien, décembre 1885.

50. LE Floch, Vincent, "Les Épidémies... ", art. cité, p. 114. 
"l'adossement de ces maisons à la colline constitue une cause permanente d'insalubrité : les murs souvent contigus ou adossés à la colline, véritables cloaques infects surtout en été [...], infiltration continuelle des eaux [...], misère, malpropreté, incurie des habitants, lits à deux étages superposés, paillasses et mauvaises couvertures, hygiène déplorable, matériels et vêtements de pêche déposés mouillés dans l'entrée, fenêtres encombrées par du poisson au séchage, poêle en fonte seulement allumé pour les repas ${ }^{51}$ ".

L'île Molène est touchée à son tour en août 1893. Chauvin, le médecin départemental des épidémies, pense que le vibrion est venu d'Angleterre, apporté par des marchands britanniques chargés d'acheter les langoustes et homards bretons. Selon le médecin de la marine Bourdon, le mal est arrivé sur l'île le 9 août, apporté par un pilote de l'île de retour du Conquet où se trouvait un malade. En septembre, on déplore 44 morts et 14 sur l'île voisine de Trielen où habitaient 23 personnes ${ }^{52}$, ceci malgré des mesures préventives importantes. Le 21 août, le patron camarétois du Saint-Jean revient à son port d'attache porteur de la maladie après avoir mouillé ses casiers dans les parages de Molène. À Sein, le médecin, le maire de l'île et le syndic font " afficher un arrêté en vertu duquel tous les bâtiments provenant du Conquet et de Molène devront être mis en observation. Cette mesure sanitaire vient d'être appliquée à un bateau de pêche qui voulait aborder à l'île ${ }^{53}$ ". Puis c'est au tour des ports de Brest et de Douarnenez d'être contaminés : le mal est repéré à Quimper le 25 septembre, puis finalement tout le Finistère littoral est atteint ${ }^{54}$. Des malades sont signalés à Audierne entre le 10 et le 23 septembre, à Plogoff entre le 13 et le 16 septembre, à Plouhinec le 18 septembre... L'évolution géographique de l'épidémie permet d'affirmer que les déplacements des marins pêcheurs sont à l'évidence à l'origine de l'épidémie qui coûte la vie à 891 Finistériens presque uniquement dans les communes maritimes : 191 décès à Brest, 92 à Saint-Pierre-Quilbignon, 113 à Lambézellec, 38 à Douarnenez, 35 à Tréboul, 18 à Quimper ${ }^{55}$. La fin de l'épidémie est officiellement déclarée le 22 octobre $1893^{56}$.

\section{La lutte contre le choléra ou le combat pour une eau propre}

Si pour de nombreux observateurs, il devient rapidement évident que la propagation du choléra s'effectue par la voie humaine et que l'intensité de l'épidémie est d'autant plus grande qu'elle survient dans des lieux sales où s'entassent des populations miséreuses, il ne faut pas oublier que la théorie de la contagion est encore dénoncée par les médecins aéristes, lesquels sont persuadés que les forts vents qui balaient les cités

51. Cité dans Monod, Henri, Le Choléra..., op. cit., p. 270.

52. Service Historique de la Défense, Marine Brest, Manuscrit santé ${ }^{\circ} 27$, rapport du médecin Bourdon, septembre 1893.

53. Le Finistère, jeudi 24 août 1893 ,

54. Arch. dép. du Finistère, 5 M 59.

55. PRISÉ, Isabelle, Deux siècles d'épidémies et d'endémies en Basse-Bretagne, 1750-1950, Thèse de doctorat en médecine, Université de Bretagne Occidentale, Brest, 1998, 205 p.

56. La Dépêche de Brest, le 22 octobre 1893. 
sardinières suffisent à chasser les miasmes. Cette théorie bien établie est parfaitement admise dans le monde entier, notamment par les sommités médicales de l'époque. En fait, il faut attendre la terrible épidémie de choléra de 1865-1866, particulièrement virulente puisqu'elle entraîne la mort de 1320 personnes environ dans le seul département du Finistère, pour que les pouvoirs publics et municipaux réagissent après avoir compris que le vibrion cholérique (que l'on ne connaît pas encore) frappe en premier lieu les ports, et plus particulièrement les ports sardiniers où l'insalubrité est totale. De là à imaginer que c'est la crasse, le manque d'hygiène et les eaux polluées qui favorisent le développement des germes pathogènes ${ }^{57}$ il n'y a qu'un pas, vite franchi par les responsables municipaux, les ingénieurs des Ponts et Chaussées et les médecins de la Marine. Pour autant, les progrès de l'hygiène publique sont bien lents et ne sont effectués que sous la pression des événements et de certains édiles particulièrement influents et déterminés. Mais pour combattre l'épidémie on s'en tient généralement à la quarantaine ${ }^{58}$.

\section{Les progrès de l'hygiène urbaine}

Les premières améliorations portent sur l'adduction d'eau douce de qualité. À Audierne, la première et unique fontaine publique est édifiée en 1844-1845, mais elle s'avère rapidement insuffisante pour répondre aux besoins de la population locale. La première conserverie est construite en 1872 par les frères Pellier qui sont particulièrement préoccupés par le manque d'eau et la mauvaise qualité des puits. Ils rédigent plusieurs lettres à la mairie où ils exposent que le manque d'eau douce " cause un préjudice considérable à leur fabrication de sardines à l'huile et ils demandent à être autorisés à établir une prise d'eau ${ }^{59}$..."

Très vite ils sont suivis par d'autres fabricants et, en 1876, on compte à Audierne une douzaine d'usines sur les bords du Goyen, un fleuve d'une saleté épouvantable alors que sur les quais, chaque été, plus de 500 chaloupes débarquent leurs poissons. Le conseil municipal se doit de réagir et présente un projet de construction d'une aiguade qui est immédiatement approuvé par les ingénieurs des Ponts et Chaussées ${ }^{60}$. Ailleurs, dans la plupart des cas, on se borne à constater les dégâts et à enterrer les morts, les conséquences du drame sont vite oubliées si bien que les autorités locales et préfectorales ne peuvent éviter le retour des épidémies.

En 1886, Henri Monod, alors préfet du Finistère, et futur directeur de l'Assistance et de l'Hygiène au ministère de l'Intérieur, n'a pas de mots assez durs pour décrire l'inaction des municipalités les plus touchées et pour lui si arriérées que la " centralisation a encore raison de leur

57. BouRdelaIs, Patrice, Les Épidémies terrassées, S.l., La Martinière, 2003, p. 139.

58. Proust, Adrien, Le Choléra, étiologie et prophylaxie, Paris, Masson, 1883, p. 176.

59. Arch. dép. du Finistère, 4 S 5, Audierne le 8 mars 1878, pétition Pellier.

60. Arch. dép. du Finistère, 2 O 40, Audierne, Arrêté préfectoral du 12 octobre 1876. 
ôter le pouvoir de faire des sottises ${ }^{61}$ ". Lors de l'épidémie de choléra de 1885-1886, il est aux avant-postes, mais il n'est guère aidé dans sa tâche quotidienne :

"Sur le littoral, les pêcheurs qui ont été particulièrement frappés dans notre épidémie mènent une existence misérable [...] il est inutile de démontrer ici que les autorités locales n'ont presque rien fait pour permettre à l'administration préfectorale de prendre à temps des mesures de défense effectives $^{62}$.

Son jugement bien acerbe est pourtant justifié à l'égard des conseillers municipaux; toutefois il faut remarquer que toutes les villes ne restent pas inactives. Audierne, encore une fois, décide la construction " de deux aqueducs à établir sur les quais". Il s'agit en fait de canalisations en fonte reliant une source du plateau au port; ainsi de l'eau potable est disponible en plus grande quantité pour les usines, les pêcheurs et les ouvrières. Peut-être estil bon de rappeler que la cité est alors dirigée par Delécluse, conserveur, et que le conseil municipal compte de nombreux fabricants, comme Louarne ou Herpe. Au Guilvinec, la mairie décide la construction de deux puits équipés de pompes et "l'installation de cabinets d'aisance aux écoles " qui en étaient jusqu'alors dépourvues ${ }^{63}$.

Dix ans plus tard, la situation n'a guère évolué quand on apprend qu'à Tréboul " les marins n'ont pas de fosses fixes étanches et quelques-uns seulement des tinettes mobiles ${ }^{64}$ "; qu'à Audierne, "le régime déplorable des fosses est encore le plus répandu ${ }^{65}$ ». On comprend mieux que les attaques microbiennes et virales s'avèrent redoutables pour les hommes. Le vibrion du choléra, découvert par Robert Koch depuis 1884, est retrouvé en avril 1894 dans l'eau de la fontaine publique du bourg du Guilvinec, année où l'épidémie est particulièrement meurtrière ${ }^{66}$. À Camaret, c'est le village du Notic où vivent les pêcheurs et les ouvrières d'usine qui est le plus durement frappé par l'épidémie de choléra en août $1893^{67}$. Pour éradiquer le mal on évacue des quartiers entiers afin de désinfecter leurs masures misérables en les badigeonnant de chaux. L'armée prête son concours et des tentes pour servir à assurer le logement provisoire des pêcheurs à Audierne et au Guilvinec " pendant qu'il

61. Arch. dép. du Finistère, 5 M 56. Monod, Henri, Notes pour Histoire d'une épidémie, Quimper, 1886.

62. Monod, Henri, Le Choléra..., op. cit., p. 51.

63. Arch. dép. du Finistère, 2 O 539, Délibération du conseil municipal du Guilvinec, le 14 août 1887.

64. Arch. dép. du Finistère, 5 M 60. Brest, le 8 février 1894, le médecin-chef des épidémies.

65. Archives de la Direction Départementale de l'Équipement de Quimper, Quimper, le 11 mai 1892, l'ingénieur en chef à $M$. Bechman, ingénieur en chef et vice-président du Comité de Médecine publique et d'Hygiène professionnelle.

66. Service Historique de la Défense, Marine Brest, 3 P 2-7, Quimper, le 5 avril 1894, le commissaire de l'Inscription maritime.

67. Arch. dép. du Finistère, 2 Z 40, Camaret, le 12 novembre 1893, registre des délibérations du conseil municipal. 
sera procédé à la désinfection de leurs logements dans ces deux localités ${ }^{68}$ " par des fumigations de soufre.

Des subventions sont votées en janvier 1894 pour permettre le pavement ou la couverture de macadam des rues du quartier du Lin à BeuzecConcq ${ }^{69}$; de même, la commune du Guilvinec débloque plus de 5000 francs pour réaliser le pavage des rues principales, afin de faciliter l'écoulement des eaux usées ou pluviales ${ }^{70}$. Les conserveurs eux-mêmes agissent parfois dans le même sens; le gérant de l'usine Philippe et Canaud de l'Île-Tudy offre 300 francs à la municipalité pour améliorer l'aménagement du bourg ${ }^{71}$, car si les épidémies restent redoutables pour les hommes, elles ne le sont pas moins pour les affaires, et les conséquences économiques du choléra ne sont pas secondaires, loin de là. Les épidémies désorganisent la vie commerciale des cités sardinières car les habitants les plus riches, les conserveurs, s'empressent de fuir les lieux infestés abandonnant aussi la production. Les exportations connaissent de sérieuses difficultés; en mai 1894, le consul de Suède et de Norvège en poste à Nantes s'inquiète de la propagation de la maladie et interdit toute sortie de conserves vers la Scandinavie car il craint la contagion. Quelques mois plus tard, il interroge les préfets des départements concernés pour connaître la situation sur l'épidémie :

" J'ai lieu d'espérer qu'aujourd'hui elle est entièrement terminée. Néanmoins pour obtenir la levée des mesures préservatrices prises en Suède et en Norvège contre les provenances du Finistère j'aurais besoin d'un avis officiel à cet égard ${ }^{72}$."

Les conserveurs ont beau rappeler au consul que les sardines sont ébouillantées et que les germes disparaissent lors de cette opération, notre Scandinave, sans doute peu familiarisé avec la nouvelle théorie pasteurienne, n'en démord pas. Les boîtes ne repartiront vers le nord de l'Europe qu'après l'épidémie terminée. Dans ces conditions il est certain que les usiniers prennent conscience des problèmes avec grand intérêt et que leurs interventions dans les séances des conseils municipaux sont plus nombreuses et plus appuyées : il faut surveiller de près les eaux potables et améliorer l'assainissement des réseaux, à tout prix. La grande enquête nationale menée sous la conduite de l'ingénieur en chef des Ponts et Chaussées, Bechman, président du Comité d'hygiène urbaine et rurale, est édifiante et ne peut que convaincre les derniers sceptiques ${ }^{73}$. Au nombre des villes les

68. Arch. dép. du Finistère, 5 M 60, Brest, le premier mars 1894, le médecin de la marine Le Jollec.

69. Arch. dép. du Finistère, 5 M 58-59, Quimper, séance du conseil général du 3 janvier 1894.

70. Arch. dép. du Finistère, 5 M 56, Le Guilvinec, le 19 février 1886. Cinq nouveaux puits sont creusés en 1894.

71. Arch. dép. du Finistère, 2 O 539. Délibération du conseil municipal, février 1894.

72. Arch. dép. du Finistère, 5 M 56, Nantes, le 17 août 1894, le consul de Suède et de Norvège au préfet du Finistère.

73. BECHMAN, Georges, Enquête statistique sur l'hygiène urbaine dans les villes françaises. Paris, Bulletin de la Société de Médecine Publique, 1892. Comme le soulignent Murard et 
plus polluées, on retrouve Douarnenez et Concarneau ${ }^{74}$. À Douarnenez, où vivent plus de 10000 habitants, durant l'été 1897 le volume d'eau « qui a pu être distribué est descendu à 1 litre 7 par habitant et par 24 heures ${ }^{75}$ ". Au Guilvinec, la population ne dispose que d'un bassin mal isolé et alimenté par un puits peu profond... Les causes de la rapidité et de la puissance de la contagion sont réunies.

L'enquête Bechman a non seulement le mérite d'alerter l'opinion publique nationale, mais aussi celui de préparer les actions futures. Pour faciliter les travaux, une circulaire ministérielle très simple à compléter est publiée pour que chaque municipalité puisse aisément présenter dans un cadre clair des projets concrets ${ }^{76}$. L'action d'Armand Considère et d'Henri Willotte, respectivement ingénieurs en chef des ponts et chaussées du Finistère et du Morbihan $^{77}$, est aussi à souligner car ils deviennent les chantres de l'hygiène publique dans leur département; ils se dépensent sans compter pour que les municipalités votent les crédits nécessaires et pour que les projets aboutissent rapidement. Ils sont fortement épaulés dans cette action par les préfectures maritimes de Brest et de Lorient qui désirent l'assainissement de tous les ports de Bretagne, le vivier des équipages de la Flotte. Dès lors, les bureaux des préfectures de Quimper et de Vannes sont submergés de demandes de travaux dès janvier 1893. Le préfet du Finistère se plaint même de ne pas disposer du personnel nécessaire pour l'étude des dossiers ou la surveillance des opérations, généralement des conducteurs des Ponts et Chaussées. On manque aussi de « tubes et flacons stérilisés nécessaires pour les prélèvements d'échantillons d'eau destinée à l'alimentation ${ }^{78}$ ". Pourtant le chantier d'adduction de l'eau potable est commencé en mars 1895 à Camaret $^{79}$. Le conseil départemental d'hygiène publique insiste aussi pour qu'un réservoir soit construit à l'Île-Tudy le plus rapidement ${ }^{80}$ et les travaux

ZylBerman, (L'Hygiène dans la République, op. cit., Paris, 1996), l'enquête n'est pas menée par le ministère lui-même, signe assez criant de la faiblesse de la direction concernée. Il s'agit pour ce comité " d'établir les premiers éléments d'une statistique des distributions d'eau en France qui n'existe même pas encore à l'état rudimentaire ". Le questionnaire est adressé à 702 villes dont la population est supérieure à 5000 habitants ou ayant une garnison, ou encore aux stations balnéaires.

74. Arch. nat., F8/188, Police sanitaire. Enquête ministérielle sur l'alimentation en eau potable des villes et des communes, 1892 (Eure-Finistère).

75. Archives de la Direction Départementale de l'Équipement de Quimper, Paris, le 22 mars 1899, l'ingénieur en chef Koziowicz.

76. Circulaire du 23 juillet 1892.

77. Armand Gabriel Considère, né à Pont-sur-Saône en juin 1848, est ingénieur en chef du Finistère de décembre 1885 à octobre 1901. Henri Willotte, né en février 1854, est ingénieur ordinaire attaché au ministère de la Marine à Brest de 1882 à 1894 puis il devient ingénieur en chef du Morbihan de septembre 1894 à octobre 1901, puis du Finistère de novembre 1901 à février 1909. Il meurt à Brest le 14 décembre 1938.

78. Archives de la Direction Départementale de l'Équipement de Quimper, Quimper, le 22 février 1895, le préfet du Finistère.

79. Archives de la Direction Départementale de l'Équipement de Quimper, Quimper le 24 février 1894, le préfet à l'ingénieur en chef des Ponts et Chaussées.

80. Arch. dép. du Finistère, 5 M 27. Quimper, séance de juin 1894. 
sont entrepris en juillet $1895^{81}$. À Audierne, en février 1896, le conseil municipal décide une vaste opération d'assainissement : il vote les crédits nécessaires pour la création de " canalisations d'eaux potables " et envisage le repavage de la place du marché et de la grand-rue,

"Considérant que l'eau est disponible en quantité insuffisante dans la cité surtout l'été pendant la saison de pêche de la sardine car alors plus de 800 bateaux étrangers se trouvent parfois dans le port ${ }^{82}$."

Bien sûr, le conseil départemental d'hygiène encourage ces initiatives. À la même époque, les communes du Guilvinec et de Penmarc'h déposent leurs projets d'alimentation en eau potable. Les chantiers commencent timidement à Douarnenez et à Concarneau, mais ne sont achevés que quinze ans plus tard alors que les égouts se multiplient et se " déversent sur la plage sans épuration ${ }^{83}$ "! Mais il faut souligner, à leur corps défendant, que les maires n'ont bien souvent pas les moyens financiers d'entreprendre de telles opérations complexes et toujours très lourdes pour les budgets communaux de l'époque. Pour la commune de l'Île-Tudy la facture s'élève à plus de 23000 francs, soit plus du double du budget annuel municipal, pour la mise en place de la canalisation en fonte, la construction d'un lavoir et de deux bornes-fontaines. Les travaux d'Audierne s'élèvent à 12000 francs et la commune ne peut les faire exécuter «ni avec ses revenus ordinaires ni avec une imposition extraordinaire dont le centime serait trop élevé et que d'ailleurs les habitants ne veulent point ${ }^{84}$ ". De même, les travaux de distribution d'eau prévus par la municipalité de Douarnenez doivent revenir à 170000 francs et bien évidemment la ville ne dispose pas de cette somme. Il est d'abord fait appel au préfet maritime pour qu'il débloque les aides nécessaires mais le ministre de la Marine ne peut donner satisfaction à la demande. Enfin, la demande de subventions à ouvrir sur les fonds du Pari Mutuel ${ }^{85}$ est acceptée et le chantier peut commencer ${ }^{86}$. Ce manque de fonds explique en grande partie les atermoiements des cités sardinières, et d'ailleurs de toutes les autres villes. Pourtant, tous les services concernés de l'État ne

81. Service Historique de la Défense, Marine Brest, Quimper, le 12 juillet 1895, le commissaire de l'Inscription maritime.

82. Arch. dép. du Finistère, 2 O 40, Délibérations du conseil municipal d'Audierne, le 2 février 1896.

83. Arch. dép. du Finistère, 5 M 103. Quimper, le 21 novembre 1912, l'inspecteur départemental d'hygiène.

84. Arch. dép. du Finistère, 2 O 40, Délibération du conseil municipal d'Audierne, le 6 avril 1896.

85. Les subventions allouées aux communes ou les prêts accordés sont prélevés sur les fonds du Pari Mutuel sur Hippodrome, organisme légalisé en juin 1891. L'argent des parieurs est utilisé pour l'amélioration de l'élevage des chevaux, pour rembourser les gagnants et, enfin, aider les communes à construire leurs réseaux d'eau potable. La subvention accordée reste faible et n'a qu'un caractère d'encouragement.

86. Arch. dép. du Finistère, 2 O 378, Quimper, le 24 novembre 1896, autorisation préfectorale. 
cessent d'intervenir pour que les réalisations aboutissent " dans les plus brefs délais ${ }^{87}$ ".

Enfin, il ne faut pas mésestimer certaines résistances particulières, exprimées notamment lors des enquête d'utilité publique. Dans leur ensemble, les propriétaires, dans les grandes villes comme à la campagne, refusent les projets, redoutant des frais et des impositions supplémentaires $^{88}$. Il n'est pas rare de constater que des personnages importants des cités concernées ne veulent pas entendre parler des projets d'assainissement jugés beaucoup trop onéreux pour les quelques habitants soumis à l'impôt. En juin 1895, la ville de Douarnenez présente un projet ambitieux d'alimentation en eau potable. L'abbé Pennamen, frère et fils de conserveur, contribuable et électeur dans cette cité, intervient pour dénoncer le projet selon une conception de la charité chrétienne bien personnelle :

«Au point de vue du principe lui-même nulle opposition. L'eau est notablement insuffisante en été. Cependant il sera permis de m'étonner que la population qui ne paie de contributions d'aucune sorte ait de l'eau à gaspiller. Cela je l'ai vu, en été, laissant couler le robinet sur un baril plein d'eau pendant que le marin à qui appartenait le baril passait dans une auberge à dix pas de là une dizaine de minutes à absorber de l'eau-de-vie et à débiter des sornettes tandis que la population qui paie toutes les contributions et assure les services de la ville est complètement privée d'eau [...] Cela vaut-il la dépense projetée? il est permis d'en douter ${ }^{89}$."

Le conserveur Le Floc'h est du même avis que son curé et maintient que c'est un leurre de croire que la ville peut tirer un bénéfice quelconque de la vente de l'eau car la population de Douarnenez est " composée pour les $9 / 10^{\mathrm{e}}$ de marins et d'ouvriers qui certainement n'achèteront rien ${ }^{90}$ ". De toute manière, les mesures prises pour améliorer la situation sanitaire restent dispersées, voire dérisoires, à l'échelle des départements, surtout à nos yeux aujourd'hui. Un nouvel espoir est entrevu après le vote de la loi sanitaire du 15 février 1902, dite loi Siegfried, acceptée après plus de dix ans d'efforts pour la porter sur l'autel parlementaire. On espérait tenir les propriétaires et les maires, leur imposer les dépenses nécessaires pour l'adduction d'eau potable, son évacuation et son traitement. Un corps d'inspecteurs départementaux d'hygiène était prévu pour surveiller les municipalités récalcitrantes. Un bureau d'hygiène devait voir le jour dans toutes les villes de plus de 20000 habitants (seulement!), mais c'est le maire et son conseil, lesquels forment dans les cités sardinières une grande réunion de famille, qui nomment le chef du service municipal d'hygiène et votent les crédits de fonctionnement de son bureau; c'est le maire qui décide des

87. Archives de la Direction Départementale de l'Équipement de Quimper, Paris, le 16 juin 1894, le ministre des travaux publics à l'ingénieur en chef du Finistère.

88. Goubert, Jean-Pierre, La Conquête de l'eau, Paris, Laffont, 1986, p. 203.

89. Arch. dép. du Finistère, 2 O 378, Douarnenez, le 23 décembre 1895, enquête d'utilité publique.

90. Arch. dép. du Finistère, 2 O 378, Douarnenez, le 25 décembre 1895. 
crédits d'amélioration sanitaire; c'est le maire qui organise les éventuels chantiers d'assainissement.

\section{De l'eau potable pour tous}

Dans un premier temps, pour protéger un tant soit peu les populations et sans débourser trop, il convient de s'intéresser aux sources, et plus généralement aux eaux d'alimentation, puisqu'il est dorénavant acquis que c'est une eau de boisson souillée qui est à l'origine des épidémies. L'eau est sale, les habitants le savent, "ils le voient; ils n'en restent pas moins très indifférents et boivent philosophiquement une eau qui ne leur fera du mal que si la destinée en a ainsi ordonné91 ! " En application de la loi de 1902, il est recommandé aux municipalités de tenir compte de la notion de zone protégée autour des surfaces de captation d'eau. À Tréboul, une enquête est menée entre le 27 juillet et le 3 août 1902 pour apprécier la qualité des sources; il est décidé d'établir un périmètre de protection de 50 mètres de diamètre autour des points d'eau et c'est tout ${ }^{92}$ ! En fait, les ingénieurs des Ponts surveillent de près ces projets et toujours ils préconisent d'élargir au maximum les zones protégées. Analysée, surveillée, testée, l'eau est offerte aux demandeurs toujours plus nombreux dans des conditions de confort et de sécurité qui s'améliorent aussi ${ }^{93}$. La notion de veille sanitaire commence à prendre un sens; les préfets, de leur côté, ne cessent de présenter des rapports alarmistes pour hâter les réalisations. À les lire, la situation est toujours aussi préoccupante sur le littoral surpeuplé de la Bretagne méridionale :

«[D]ans le Finistère existent plusieurs agglomérations importantes très insuffisamment ou pas du tout pourvues d'eau potable. Le voisinage immédiat de la mer ne permet guère d'y rechercher l'eau de source au moyen de puits qui ne donneraient d'ailleurs qu'une quantité d'eau infime par rapport aux besoins des grandes agglomérations. Il en résulte des inconvénients multiples au point de vue de la salubrité publique ${ }^{94}$."

Pourtant les demandes ne cessent d'être toujours plus nombreuses et plus pressantes, mais l'insuffisance des fonds du " Pari mutuel " ne permet pas de donner satisfaction à toutes les municipalités. Tous les textes et enquêtes officielles d'époque nous confirment que les progrès réalisés pour améliorer la situation sanitaire des ports de Bretagne et de Vendée demeurent d'une lenteur désespérante, mais ils sont effectués ${ }^{95}$.

91. Monod, Henri, Le Choléra..., op. cit., p. 58.

92. Arch. dép. du Finistère, 5 M 102. Séance du conseil d'hygiène, août 1902.

93. GouBert, Jean-Pierre, La Conquête de l'eau..., op. cit., p. 46.

94. Arch. dép. du Finistère, 1 M 133, Quimper, juillet 1910, rapport du préfet du Finistère.

95. Arch. nat., F8/217, Enquête (circulaire du 5 novembre 1912) sur les conditions d'alimentation en eau potable et évacuation des eaux usées, Finistère et Arch. nat., F8/220, Morbihan. 


\section{Le choléra vaincu}

À la veille de la Grande Guerre, il est évident que l'éducation sanitaire a fait de gros progrès au sein des équipages militaires et les habitudes acquises sur les vaisseaux de l'État sont souvent poursuivies dans les familles. La diffusion des traités d'hygiène navale prouve indubitablement que les consignes, à défaut d'être respectées, sont connues ${ }^{96}$. Les Écoles de pêche apprennent aussi aux marins à reconnaître les premiers symptômes inquiétants ${ }^{97}$. Quoi qu'il en soit, toutes ces actions ne suffisent pas à expliquer la forte baisse de la mortalité et dans ces conditions il faut bien admettre que les progrès sanitaires restent une des causes principales de cette situation générale d'amélioration de la condition humaine. La corrélation entre les travaux d'assainissement et d'adduction et la baisse de la mortalité est évidente. Bien sûr, les chantiers réalisés apparaissent à nos yeux bien sommaires mais ils suffisent souvent à franchir l'étape initiale primordiale : on passe ainsi de l'absence totale d'équipement aux premiers balbutiements d'une nouvelle société. L'eau courante, propre et potable parvient à la veille de la Grande Guerre dans toutes les communes sardinières du littoral breton; sale, elle est évacuée à l'extérieur des murs; ces opérations améliorent les conditions de vie et par conséquent la santé des consommateurs, mais surtout modifie leur comportement, leurs mœurs, et transforme les esprits ${ }^{98}$. "Un abreuvoir, un lavoir, une pompe, une dérivation, des conduites en fonte plus ramifiées, quelques bornes-fontaines bien situées, un raccordement au réseau... ", tels sont les travaux entrepris à une échelle modeste mais réelle ${ }^{99}$ et qui ont modifié rapidement les effets de surmortalité dans les cités sardinières et bien avant que les campagnes et les petites villes françaises ne soient équipées. Certes, il est difficile de se livrer à des analyses précises concernant le recul du choléra, de la typhoïde et de la dysenterie, mais on peut clairement observer la concordance chronologique entre la mise en place des premières politiques sanitaires et la diminution des maladies infectieuses transmises par l'eau ${ }^{100}$. Si les travaux entrepris sur le littoral breton sont tardifs et incomplets, il convient de souligner qu'ils sont accomplis précocement à l'échelle nationale et que les résultats espérés, et obtenus, prouvent leur réelle efficacité, contrairement à l'opinion généralement admise.

En 1895 est construit à Brest le laboratoire de bactériologie à l'hôpital des Armées sous la responsabilité du médecin chef Auffret, un hygiéniste convaincu $^{101}$. Dès lors, les analyses deviennent plus efficaces. Sans attente,

96. LAVERAN, Alphonse, Traité d'hygiène militaire, Paris, Masson, 1896, XIV-896 p. LEMOINE, Georges, Traité d'hygiène militaire. Paris, Masson et Cie, 1911, xxIv-758 p.

97. Bronkhorst, Louis, Henensal, M., Lesage, R., Manuel du patron à la pêche et des candidats au brevet de patron à la pêche, Paris, SEGMC, 1926, p. 330-331.

98. Arch. nat., F8/2250, Travaux d'adduction d'eau, Finistère (1906-1931) et Arch. nat., F8/2270, Morbihan, (1895-1931)

99. GouBERT, Jean-Pierre, La Conquête de l'eau..., op. cit., p. 217.

100. Bourdelais, Patrice, Les Épidémies terrassées..., op. cit., p. 148 et suivantes.

101. Pluchon, Pierre, Histoire des médecins..., op. cit., p. 178. 
dès que les premiers soupçons s'installent, une analyse est effectuée et si la présence du vibrion est attestée dans l'eau, on peut prendre les mesures de protection très rapidement. Simultanément, l'utilisation massive de l'hypochlorite de sodium ${ }^{102}$ stimule la lutte anticholérique car le germe responsable du choléra est détruit très efficacement par ce produit chimique selon les constatations effectuées par Koch dès 1881. En 1901, deux cas de " diarrhées cholériformes, dont un suivi de décès " sont relevés à Douarnenez; en 1905 on note encore un décès à Morgat, deux cas à Brest, dans le quartier de Kéravel et plusieurs cas dans la maison d'un pêcheur du Conquet ${ }^{103}$. L'année suivante, on enregistre un décès à Brest, mais il semble bien que la maladie régresse; les progrès en matière d'hygiène urbaine semblent payants. Les épidémies de choléra sont vaincues dans la Basse-Bretagne maritime.

Entre 1850 et 1914, plus de deux cents usines de sardines à l'huile se construisent sur le littoral atlantique entre Camaret et Arcachon, dans la confusion et le désordre, au sein d'une société maritime préexistante qui subit de profondes modifications. Cette longue région côtière connaît concomitamment un formidable essor démographique, lequel entraîne de multiples bouleversements dans la structure urbaine des cités concernées, dont certaines voient le jour à cette occasion. La densification s'accompagne de perturbations sociales et sanitaires, et notamment d'une surmortalité importante, au moins durant la première période étudiée, l'expression " la plus tangible des effets d'une industrialisation mal contrôlée ${ }^{104}$ " comme dans tous les bassins industriels de l'époque. Plus que la croissance démographique, ce sont les ravages épidémiques du choléra en particulier, et la mauvaise qualité des eaux qui entraînent une modification des comportements sanitaires. Ces redoutables dangers sont dénoncés par les fabricants qui s'inquiètent de leurs conséquences économiques; les plaintes de ces derniers poussent les autorités municipales à prendre des mesures d'urgence et leur imposent d'organiser des services d'hygiène de plus en plus sûrs. Il faut croire que cette volonté d'amélioration porte ses fruits car on constate que les taux de mortalité dans toutes les villes sardinières connaissent une baisse rapide et précoce, toujours plus marquée que celles des autres villes des départements maritimes, après 1895 environ. Cette constatation demande une enquête beaucoup plus approfondie car elle ne correspond pas aux conclusions des observateurs de l'époque, souvent reprises sans vérification par les auteurs actuels. La surmortalité urbaine originelle fait place à une sous-mortalité évidente mais méconnue, au moins à l'échelle du bassin sardinier.

102. Contenue dans l'eau de Javel.

103. Arch. dép. du Finistère, 5 M 61, Choléra 1900-1924. Brest, le 10 septembre 1905, le commissaire spécial.

104. Mumford, Lewis, La Cité à travers l'histoire, Paris, Le Seuil, 1964, p. 586. 


\section{RÉSUMÉ}

Comme tous les bassins industriels, le littoral sardinier breton connaît au $\mathrm{XIX}^{\mathrm{e}}$ siècle une croissance démographique exceptionnelle, surtout par rapport aux autres régions de la province, intérieures et agricoles. Dans certaines localités, où plus de cent cinquante usines de sardines à l'huile ont été construites dans la confusion et le désordre, comme à Douarnenez, au Guilvinec, à Concarneau ou à La Turballe, le chiffre de la population urbaine triple en moins d'un demi-siècle. Tous ces ports sardiniers sont caractérisés par une hygiène publique et individuelle déplorable, résultat de la convergence spontanée entre les intérêts des usiniers, le laxisme des conseils municipaux et des comportements individuels indifférents et passifs. La crasse, la surpopulation urbaine, le manque d'eau potable sont responsables de la multiplication d'épidémies d'une rare violence, et notamment des attaques du choléra, qui ravagent la Bretagne littorale entre 1832 et 1912. Ce redoutable danger est dénoncé par les fabricants de sardines à l'huile qui s'inquiètent de ses conséquences économiques; les plaintes de ces derniers poussent les autorités municipales à prendre des mesures d'urgence et leur imposent d'organiser des services d'hygiène de plus en plus sûrs. Il faut croire d'ailleurs que cette volonté d'amélioration porte ses fruits car on constate que les taux de mortalité, dans toutes les villes sardinières, connaissent, après 1895 environ, une baisse rapide et précoce, toujours plus marquée que dans les autres villes des départements côtiers. La surmortalité urbaine fait place à une sousmortalité, évidente mais méconnue, au moins à l'échelle du bassin sardinier. Cette constatation demanderait une enquête beaucoup plus approfondie car elle ne correspond pas aux conclusions des observateurs de l'époque souvent reprises par les auteurs actuels sans vérification.

\section{ABSTRACT}

The remarkable population growth experienced by all industrialised areas also characterised the sardine-processing coastal towns in 19th-century Brittany, especially when compared with the agriculture-dependent parts of the region further inland. In small towns like Douarnenez, Le Guilvinec, Concarneau or La Turballe, where over 150 sardine-processing units were hastily built in a disorderly way, urban population trebled in less than 50 years. All such harbours specialising in the sardine business suffered from poor public and private hygiene standards, as a result of self-combining factors, namely the industrialists' interests, lax local authority stances and passive, indifferent individual behavioral patterns. Filth, urban overpopulation, lack of drinking water led to a surge in violent epidemics. Severe cholera outbreaks notably affected coastal Brittany between 1832 and 1912. The fearsome danger was denounced by tinned sardines makers worried by potential adverse economic effects. Their complaints urged local authorities to take urgent action and to impose the implementation of ever safer hygiene services. Admittedly the drive for improvement was fruitful as mortality rates swiftly decreased across all sardine-processing towns after circa 1895. They outpaced the other towns from the coastal counties. Across the sardine belt at least, urban overmortality turned into an undeniable yet little known. undermortality. This very fact is a topic for further research as it contradicts the conclusions of contemporary observers, which are often reproduced unverified in today's literature. 